\title{
Nano-plasticity of Single-Wall Carbon Nanotubes under Uniaxial Compression
}

\author{
Deepak Srivastava $^{a *}$, Madhu Menon ${ }^{b * *}$ and Kyeongjae Cho ${ }^{c * *}$ \\ ${ }^{a}$ NASA Ames Research Center, MS T27A-1, Moffett Field, CA 94035-1000 \\ ${ }^{b}$ Department of Physics and Astronomy, University of Kentucky, Lexington, KY 40506-0055 \\ ${ }^{c}$ Mechanics and Computation Division, Department of Mechanical Engineering, Stanford \\ University, CA 94305-4040
}

(April 15, 1999)

\begin{abstract}
Nano-plasticity of thin single-wall carbon nanotubes under uniaxial compression is investigated through generalized tight-binding molecular dynamics (GTBMD) and ab-initio electronic structure methods. A novel mechanism of nano-plasticity of carbon nanotubes under uniaxial compression is observed in which bonding geometry collapses from a graphitic $\left(s p^{2}\right)$ to a localized diamond like $\left(s p^{3}\right)$ reconstruction. The computed critical stress $(\approx 153 \mathrm{G} \mathrm{Pa})$ and the shape of the resulting plastic deformation is in good agreement with recent experimental observation of collapse and fracture of compressed carbon nanotubes in polymer composites.
\end{abstract}




\section{INTRODUCTION}

The discovery of carbon nanotubes (CNTs) by Iijima, $[1,2]$ and subsequent observations of CNT's unique mechanical and electronic properties have initiated intensive research on these quasi-one dimensional structures. CNTs have been identified as one of the most promising building blocks for future development of functional nano-structures. Accurate characterization of nano-mechanics in the elastic and plastic regimes, however, is highly desirable for any application in nano-composites and/or devices. As external stress is applied to nanotubes, initial linear elastic deformations are observed up to a certain critical strain beyond which nonlinear responses set in. In the non-linear response regime, locally deformed structures such as pinches, kinks and buckles have been observed in both experiments and simulations. [3-12]

Specifically, classical molecular dynamics (MD) simulations, employing Tersoff-Brenner many-body atomistic interactions, $[13,14]$ have been performed for CNTs under tensile and compressive stresses. [7,9] Both single and multi-walled CNTs are shown to be extremely elastic, well beyond the typical yield strain of $0.1 \%$ for most other materials. Surprisingly, compressed CNTs in the non-linear elastic response regime are also shown to completely recover from severe structural deformations such as localized pinches and kinks. $[7,9,12] \mathrm{It}$ is predicted, therefore, that the elastic limit of these materials would be extremely high. Under tensile strain, a plastic response behavior primarily driven by Stone-Wales (SW) bond rotation defects, [17] that generate pentagon-heptagon (5/7) pair defects in a graphene lattice, has been proposed. $[15,16]$ It is suggested that the axially compressed C.NTs would also behave similarly and deform plastically via SW defect formation mechanism. [15] The classical MD simulations of compressed nanotubes performed so far, however, show only completely elastic deformation up to $15 \%$ and higher strain for similar tubes. $[7,12,9]$ In these simulations, CNTs behave as elastic rods that pinch and buckle locally into a variety of morphological patterns that were predicted in a continuum mechanics based shell model description of the nano-mechanics. [7] 
In a recent experiment, large compressive strains were applied to CNTs dispersed in composite polymeric films. Two distinct deformation modes; sideways buckling of thick tubes, and collapse/fracture of thin tubes without any buckling, have been observed. [18] The buckling of thick tubes is in qualitative agreement with the classical MD simulation and shell model predictions of Yakobson et. al. [7]. The plastic collapse or fracture of thin tubes without any buckling, however, is contrary to the classical MD simulation results known so far. $[7,15,9]$ The compressive strain in the experiment is estimated to be larger than $5 \%$, and critical stress for inward collapse or fracture is expected to be 100-150 G Pa for thin tubes. [18]

In this Letter, we investigate the plasticity of compressed nanotubes using quantum generalized tight-binding molecular dynamics (GTBMD) and $a b$ initio electronic structure methods. We report collapse and plasticity of compressed thin nanotubes via graphitic $\left(s p^{2}\right)$ to diamond $\left(s p^{3}\right)$ like bonding reconstruction at the location of the collapse, that is driven by the relaxation of the accumulated strain energy in the uncollapsed section of the tube. The nature of the collapse is in qualitative agreement with the experimental observation of Lourie et. al. and the estimated critical stress $(\approx 153 \mathrm{G} \mathrm{Pa})$ is also within the experimentally predicted range. [18]

The molecular dynamics simulations of the compression of nanotubes are performed with a quantum GTBMD method of Menon and Subbaswamy [20] that makes explicit use of the non-orthogonality of the orbitals in treating interactions in covalent systems. The method has been found to be very reliable in obtaining good agreement with experimental results for the structural and vibrational properties of fullerenes and nanotubes. [20,21] Equilibrium geometries for small carbon clusters obtained by the GTBMD method [22] are also in good agreement with $a b$ initio results. [23]

The axial compression of an $(8,0)$ CNT is achieved by keeping the edge atoms of the tube transparent to the forces generated in the GTBMD method. The positions of the edge atoms are moved axially inward at a fixed rate to compress the nanotube. Each $1 \%$ compression in the linear response regime, and $0.25 \%$ compression near elastic limit, is accompanied by 
GTBMD relaxation of 800 steps. Keeping the strained atoms fixed, the edge atoms are then adjusted and relaxed for 200 more GTBMD steps. The GTBMD relaxation of 1000 MD steps at each strain value is sufficient to reduce the forces on atoms in the deformed region to below $0.02 \mathrm{eV} / \AA$. At the elastic limit $(12 \%$ compression for an $(8,0) \mathrm{CNT})$, the compressed tube is relaxed for 5000 GTBMD steps, during which a spontaneous plastic collapse of the tube is observed.

The compressed nanotube energetics during the collapse are also analyzed with an $a b$ initio density functional theory (DFT) using pseudo-potential method. [24] Plane wave basis sets with a cutoff energy of $40 \mathrm{Ry}$ is used for the expansion of the single electron wave functions (Kohn-Sham orbitals), and the local density approximation (LDA) is used for the exchange-correlation energy functional. Up to 36,000 plane wave basis functions are used to expand each electronic wave function for accurate energetics estimation.

The strain energy per atom is calculated as the difference in the total energy per atom of the strained and unstrained tube, and is shown in Fig. 1a, as a function of percentage strain. For comparison, we also show the strain energy calculated the same, but for classical MD simulations employing the Tersoff-Brenner model of many-body atomistic interactions. [14] Parabolic fits to two sets of data in the linear elastic response regime can be used to compute the Young's Modulus for the nanotube. The calculated value from the GTBMD method is $1.3 \mathrm{~T} \mathrm{~Pa}$ (using $3.4 \AA$ for the CNT thickness) for the $(8,0)$ nanotube considered. For low value of compressive strain $(\leq 8 \%)$, before any structural deformation occurs, the classical MD values are also in reasonably good agreement with the quantum GTBMD values as shown in Fig.1a. Significant differences, however, start to occur for compressive strain larger than $8 \%$. First, the GTBMD curve (Fig.1a) shows that the $(8,0)$ nanotube can be compressed up to $12(+/-0.25) \%$ before any structural deformation occurs. The classical MD simulation for the same nanotube, however, shows the structural deformation to start between 8 and $9 \%$. Moreover, the nature of the structural deformation in the two cases is also significantly different. The structural deformation at $12 \%$ strain (as shown in Fig.1b) in the quantum GTBMD method is completely spontaneous and leads to plastic collapse 
of the tube. On the other hand, the structural deformation in the classical MD method observed between 8 and $9 \%$ strain, resulting in the formation of symmetric-pinching mode, is completely elastic. [25]

The microscopic details and mechanism of the structural collapse are discussed next. At $12 \%$ strain, as shown in Fig.2a, structural deformation starts asymmetrically at two locations in the tube with small changes in an otherwise circular cross-section. Strain relaxation in the center (highly strained) region of the tube forces the atoms, at the locations of the deformations, to gradually collapse inward as shown in Figs.2b and c. Four-fold coordinated bonds are formed and the structure is further 'pulled' inwards by the newly formed ( $s p^{3}$ type) bonds (Fig.2d). The energetics (shown in Fig.1b) of the spontaneous inward collapse as discussed above show that there is net energy release for this process. As discussed below any local activation energy barrier is overcome by large release of strain energy in the uncollapsed section.

The structural changes during the CNT collapse are further illustrated in a radial distribution function (RDF) analysis that is shown in Figs. 3a and b. For simplicity, only the RDF peak around first neighbor shell radial distance is discussed. At $0 \%$ compression there is a single peak around equilibrium carbon-carbon ( $s p^{2}$ type) bond distance of $1.42 \AA$ in unstrained graphene sheet. At $4 \%$ compression a second peak, representing compressed bonds along the tube axis, develops at about $1.37 \AA$. The original peak with a reduced magnitude also shifts to a lower value of $1.41 \AA$. This indicates that most of the axial strain is accommodated only in the bonds parallel to the tube axis. The remaining strain has been used up in the opening of bond-angles and in increasing the tube radius as defined by the Poisson ratio. At $8 \%$ strain the second peak, representing strained bonds parallel to tube axis, shifts to about $1.33 \AA$, while the first peak representing unstrained bonds does not move much. At $12 \%$ strain the structural collapse occurs (Fig. 2d) and the second peak, representing strained bonds parallel to the tube axis, vanishes. The original peak, representing mostly the uncollapsed section, is also significantly reduced in magnitude. Many smaller peaks (like a background noise type distribution of bond lengths) in the range 1.34 
$-1.60 \AA$ are observed. This signifies a break-down of the starting (strained or unstrained) $s p^{2}$ type bonding structure.

Contributions to the over-all bond length distribution due to collapse are further analyzed in Fig.3b. Dotted line in the background shows that the uncollapsed section still preserves the two peak structure. The original peak representing non-axially aligned non-strained bonds at around $1.42 \AA$ and the strained (but split) peak around $1.36 \AA$ representing axially aligned strained bonds in the uncollapsed section. This shows that the strain in the uncollapsed section has reduced from $12 \%$ to $4 \%$. The dashed line in the middle representing the collapsed section shows a wide distribution $(1.42-1.55 \AA)$ of bond lengths. The lower edge near $1.42 \AA$ represents $s p^{2}$ type bonds of the original but collapsed graphene tube while the upper edge near $1.55 \AA$ represents compressed $s p^{3}$ type bonds of diamond like reconstruction that is shown in Fig. 2d.

The nature of the driving mechanism for the plastic failure is investigated next by examining the $a b$ initio total energy changes as the tube collapses. The collapsed part (left section in 2d.) contains six 4-fold coordinated atoms that induce a significant reduction (from $7.4 \AA$ to $6.1 \AA$ ) in the axial length of this section. The $a b$ initio energetics reveals that there is a net $3.0 \mathrm{eV}$ energy increase in the collapsed section, and a local activation barrier of about $8.4 \mathrm{eV}$ to the collapse. Comparing the energetics of the same section with the value at the beginning of the collapse leads to an effective spring constant of $\mathrm{K}=5171.6$ $\mathrm{eV}\left(\Delta E=K \epsilon^{2} / 2\right)$. This corresponds to a Young's modulus of $1.4 \mathrm{~T} \mathrm{~Pa}$ before collapse and a net length reduction of about $1.3 \AA$ due to the collapse. The accumulated axial strain in the uncollapsed section, on the other hand, is simultaneously reduced, providing the driving force for the observed mechanism as well. The estimated strain energy release in the uncollapsed section $(\approx 50 \mathrm{eV}$, estimated from the effective spring constant) for the uncollapsed section is large enough to overcome the estimated local activation barrier and deposit an extra strain energy of magnitude $3 \mathrm{eV}$ in the compressed collapsed section. The remaining released strain energy of the uncollapsed section is dissipated in the form of heat and is 
removed by the GTBMD energy minimization process. Thus there is a net strain energy release through the local collpasing process.

Even though our analysis is based on simulations for short length CNTs (320 atoms containing 20 units of $\mathrm{N}=16$ each), we can generalize it to longer length CNTs. Assuming that the CNT could experience a similar collapse, and that the collapsed part is compressed by the same $(1.3 \AA)$ amount with an equivalent release of strain in the uncollapsed part, we can estimate the strain energy released for a longer uncollapsed part. The strain on the uncollapsed part is $12 \%$ before the collapse and is reduced by $1.3 / \mathrm{N} / 8.7$ due to collapse elsewhere. Where there are $4 \mathrm{~N}$ equivalent sections of nanotubes in the middle with each section measuring $8.7 \AA$ before the collapse. The residual strain on the uncollapsed part is $\epsilon=0.12-1.3 / 8.7 / \mathrm{N}$, and the net strain energy change is $-37.2^{*}(2.5 \mathrm{~N}-1.6) / \mathrm{N} \mathrm{eV}$, where we have used the calculated spring constant given earlier. The longer $(8,0)$ CNT, in the large N limit, releases $93 \mathrm{eV}$ per each plastic collapse of the nature described above. The net strain energy release $(93 \mathrm{eV})$ in the uncollapsed section is thus still large enough to overcome any local activation energy barriers for collapse or even produce fracture without any buckling. [18]

In the above analysis we have assumed that the mechanism of the collapse remains unchanged as the tube length increases. A simple estimation of the Euler buckling stress for the length $(\approx 4 \mathrm{~nm})$ of the tube used in this simulation shows that the critical stress needed for buckling or other morphological deformations is about twice as much as the value at which the tube collapsed in our work. For longer tubes, however, the needed critical stress for Euler buckling will be less, and could compete with the above collapsing or plastic deformation mechanism. Similarly, a larger diameter tube of similar length could also be compressed to first undergo Euler buckling before neighboring carbon atoms start to interact strongly causing the inward collapse of the structure with $s p^{2}$ to $s p^{3}$ type reconstruction. This explains the observation of both buckling of thick tubes and collapse/fracture without buckling of thin tubes in experiments. [18] The critical lengths and diameters of the tube, above which buckling or other morphological deformations might dominate over the 
collapsing mechanism, will be investigated in future.

In summary, we have presented a novel nano-plastic mechanism of compressed nanotubes where local tetrahedral bonds $\left(s p^{3}\right)$ of carbon atoms form at the location of the collapse. This is also reminiscent of graphitic to diamond-like phase transitions observed in high compression pressure cells $(\approx 150 \mathrm{G} \mathrm{Pa})$ in the core of irradiated and annealed bucky onion. [19]. In our case, the mechanism is driven by the release of large amount of accumulated strain in the uncollapsed section through structural relaxation. The computed critical stress $(\approx 153$ $\mathrm{G} \mathrm{Pa}$ for $12 \%$ compressed tube) is in good agreement with the the experimentally estimated range of values reported by Lourie et. al., for thin nanotubes. [18] Most significantly, our work considerably lowers the elastic limit for free standing thin compressed nanotubes (to about $12 \%$ ) as compared to the $15 \%$ and higher values computed by classical MD atomistic simulations. [7,9] Investigations of the effect of tube diameter and chirality on the elastic limit and the above mechanism of plasticity are currently underway.

\section{ACKNOWLEDGMENT}

Part of this work (DS) is supported by MRJ under NASA contract NAS2-14303. MM acknowledges support by NSF grant OSR 94-52895, and by the University of Kentucky Center for Computational Sciences. KC's work is supported by Stanford's Terman Award from the Packard Foundation and the NSF-MRSEC. The GTBMD simulations were performed on a SGI Origin2000 at NAS at NASA Ames Research Center, and $a b$ initio computations were performed on a CrayT90 allocated through the NPACI Grant "Nanoscale Materials Simulations." 


\section{REFERENCES}

*e-mail: deepak@nas.nasa.gov

** e-mail: super250@pop.uky.edu

*** e-mail: kjcho@stanford.edu

[1] S. Iijima, Nature 354, 56 (1991).

[2] S. Iijima, T. Ichihashi and Y. Ando, Nature 356, 776 (1992).

[3] S. Iijima, C. Brabec, A. Maiti, and J. Bernholc, J. Chem. Phys. 1042089 (1996).

[4] J. Despres, E. Daguerre, K. Lafdi, Carbon 3387 (1995).

[5] N. Chopra, L. Benedict, V. Crespi, M. L. Cohen, S. G. Louie, A. Zettl, Nature 377, 135 (1995).

[6] R. Ruoff and D. Lorents, Bull. Am. Phys. Soc. 40,173 (1995).

[7] B. I. Yakobson, C. J. Brabec, and J. Bernholc, Phys. Rev. Lett. 762511 (1996).

[8] M. R. Falvo, G. J. Clary, R. M. Taylor II, V. Chi, F. P. Brooks Jr., S. Washburn, R. Superfine, Nature 389, 582 (1997).

[9] D. Srivastava and S. Barnard, Proc. IEEE Supercomputing '97 (SC '97), CDROM version (1997).

[10] W. H. Knechtel, G. S. Dusberg, and W. J. Blau, Appl. Phys. Lett. 731961 (1998).

[11] W. Clauss, D. J. Bergeron and A. T. Jhonson, Phys. Rev. B58 4266 (1998).

[12] J. Bernholc, C. Brabec, M. Buongiorno, A. Maiti, B. I. Yakobson, Appl. Phys. A67, 39 (1998) and references there in.

[13] J. Tersoff, Phys. Rev. Lett. 612879 (1988).

[14] D. W. Brenner, Phys. Rev. B42 9458 (1990). 
[15] B. I. Yakobson, Appl. Phys. Lett. 72, 918 (1998).

[16] P. Zhang, P. E. Lammert and V. H. Crespi, Phys. Rev. Lett. 8153461998.

[17] A. J. Stone and D. J. Wales, Chem. Phys. Lett. 128501 (1986).

[18] O. Lourie, D. M. Cox, H. D. Wagner, Phys. Rev. Lett. 81, 1638 (1998).

[19] F. Banhart and P. M. Ajayan, Nature 382, 433 (1996).

[20] M. Menon, E. Richter and K. R. Subbaswamy, J. Chem. Phys. 104, 5875 (1996).

[21] A. M. Rao, E. Richter, S. Bandow, B. Chase, P. C. Eklund, K. A. Williams, S. Fang, K. R. Subbaswamy, M. Menon, A. Thess, R. E. Smalley, G. Dresselhaus, and M. S. Dresselhouse, Science 275187 (1997).

[22] M. Menon and K.R. Subbaswamy, Phys. Rev. B 55, 9231 (1997).

[23] K. Raghavachari and J. S. Binkley, J. Chem. Phys., 87, (1987) 2191.

[24] For more details see a review article: M.C. Payne et al., Rev. Mod. Phys. 64, 1045 (1992).

[25] This is similar to the observation reported also by Yakobson et. al. in Ref. 7 . 


\section{FIGURES}

FIG. 1. (a) Strain energy as a function strain in an axially compressed $(8,0)$ nanotube. Filled circles are for compression computed with the quantum GTBMD method where as stars are for the values computed with classical MD method. Inset (b) shows the strain energy minimization at $12 \%$ strain as a function of number of GTBMD relaxation steps.

FIG. 2. Plastic collapse of of $12 \%$ compressed $(8,0)$ carbon nanotube shown at points $1,2,3$ and 4 in $\mathrm{a}, \mathrm{b}, \mathrm{c}$, and $\mathrm{d}$, respectively.

FIG. 3. Radial distribution function (RDF) near first neighbor shell as a function of radial distance. (a) RDF at $0,4,8$, and $12 \%$ compressed and GTBMD energy minimized tube, and (b) contributions to $12 \%$ compressed tube RDF from central uncollapsed and two collapsed sections of the tube. 


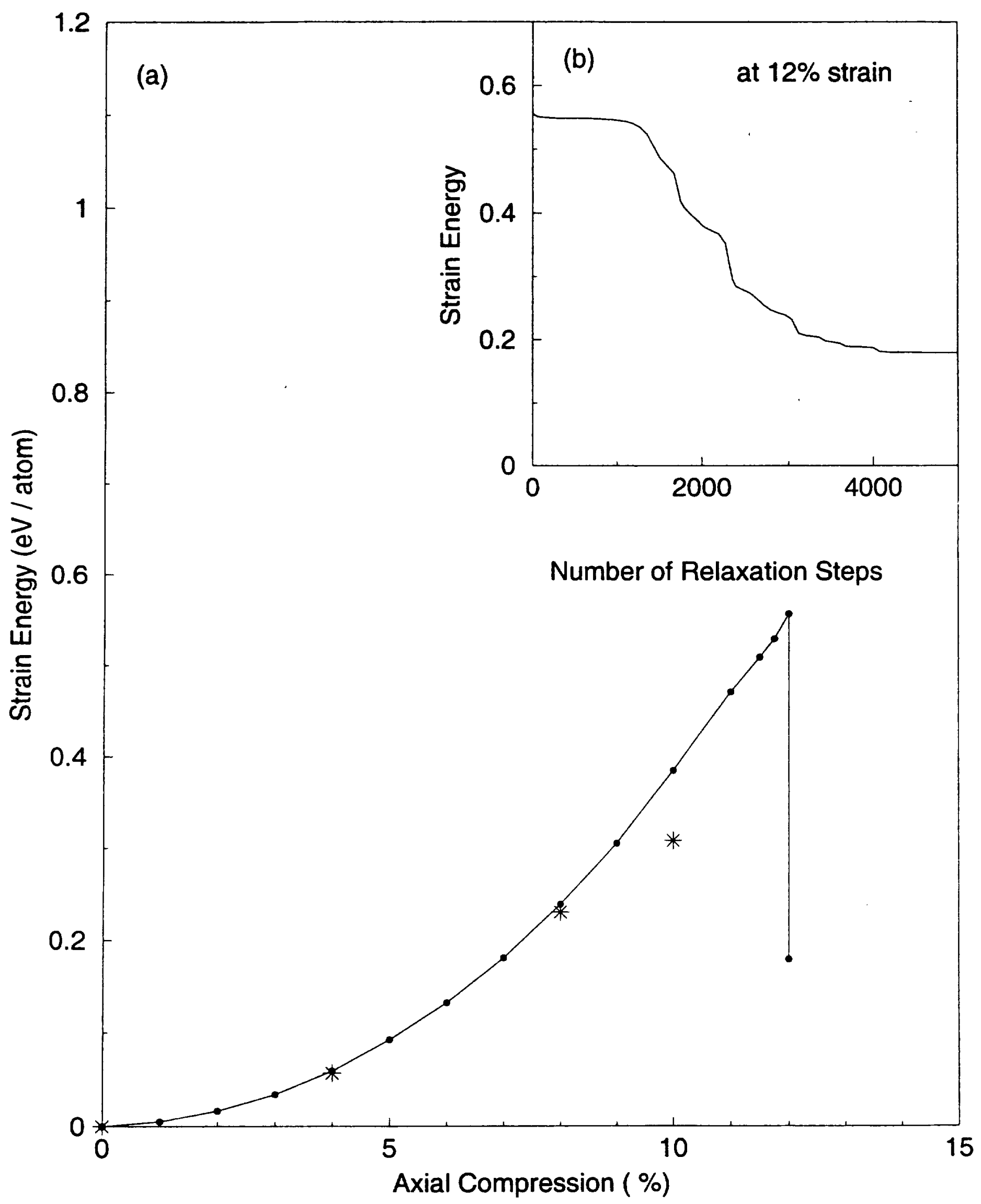



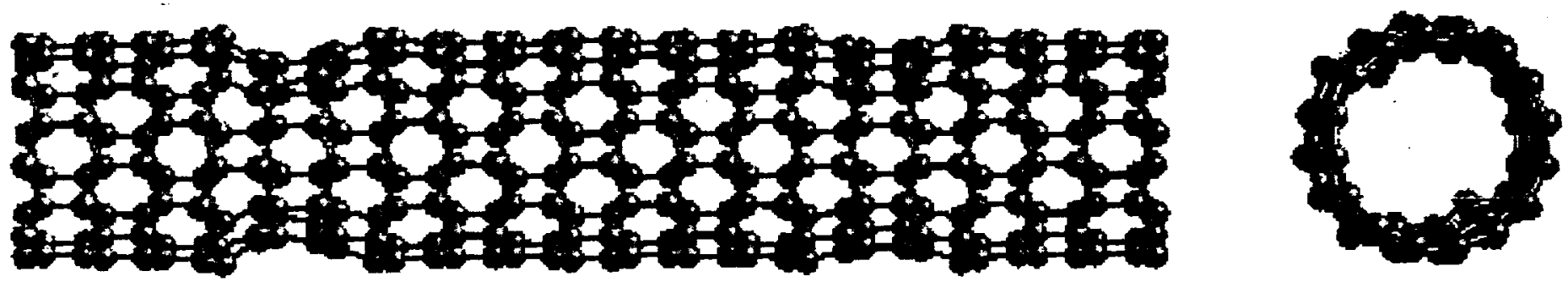

(a)
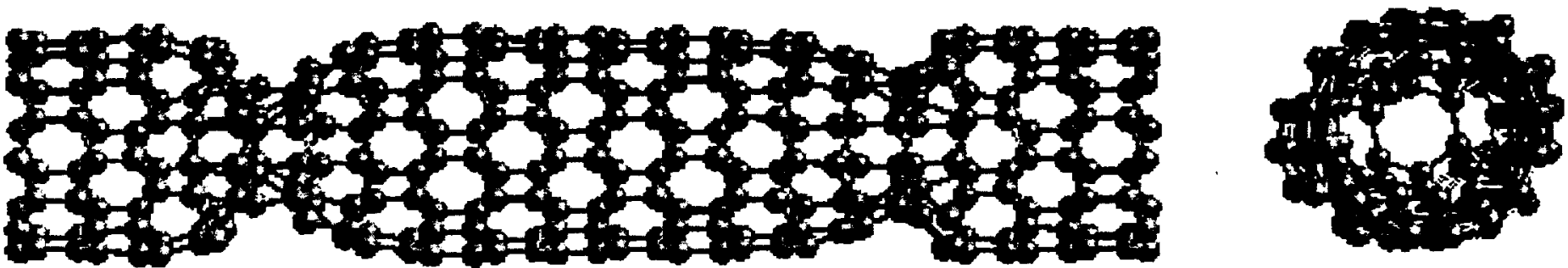

(b)
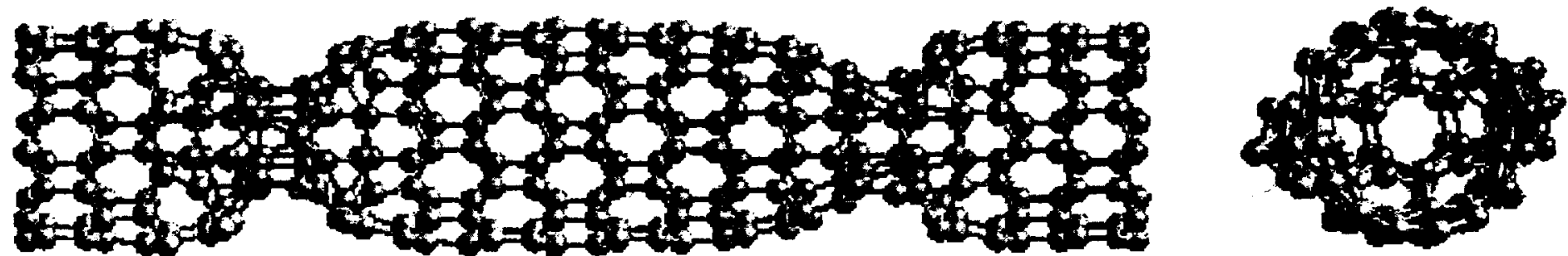

(c)

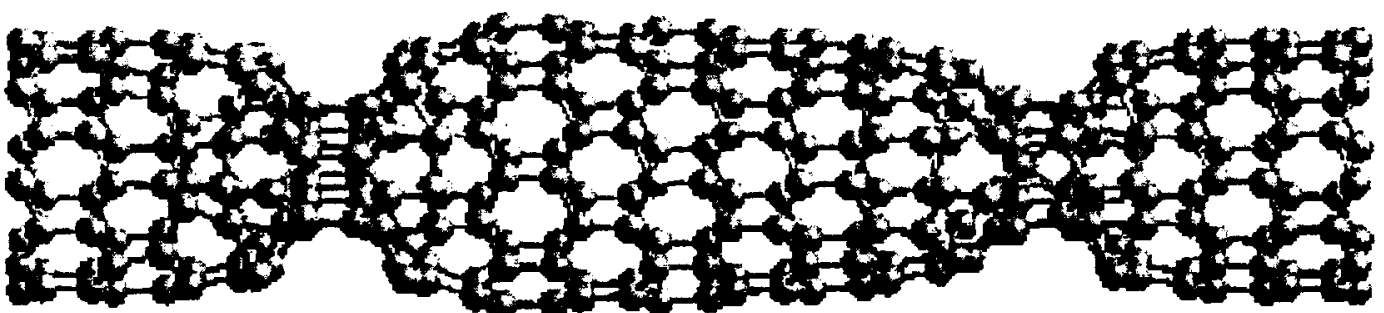

(d)

radial view

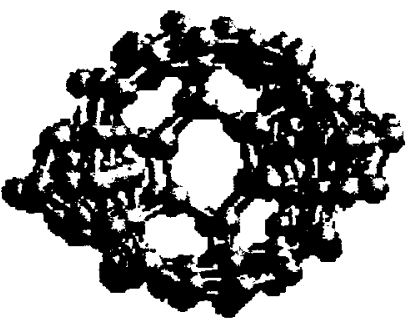

axial view 


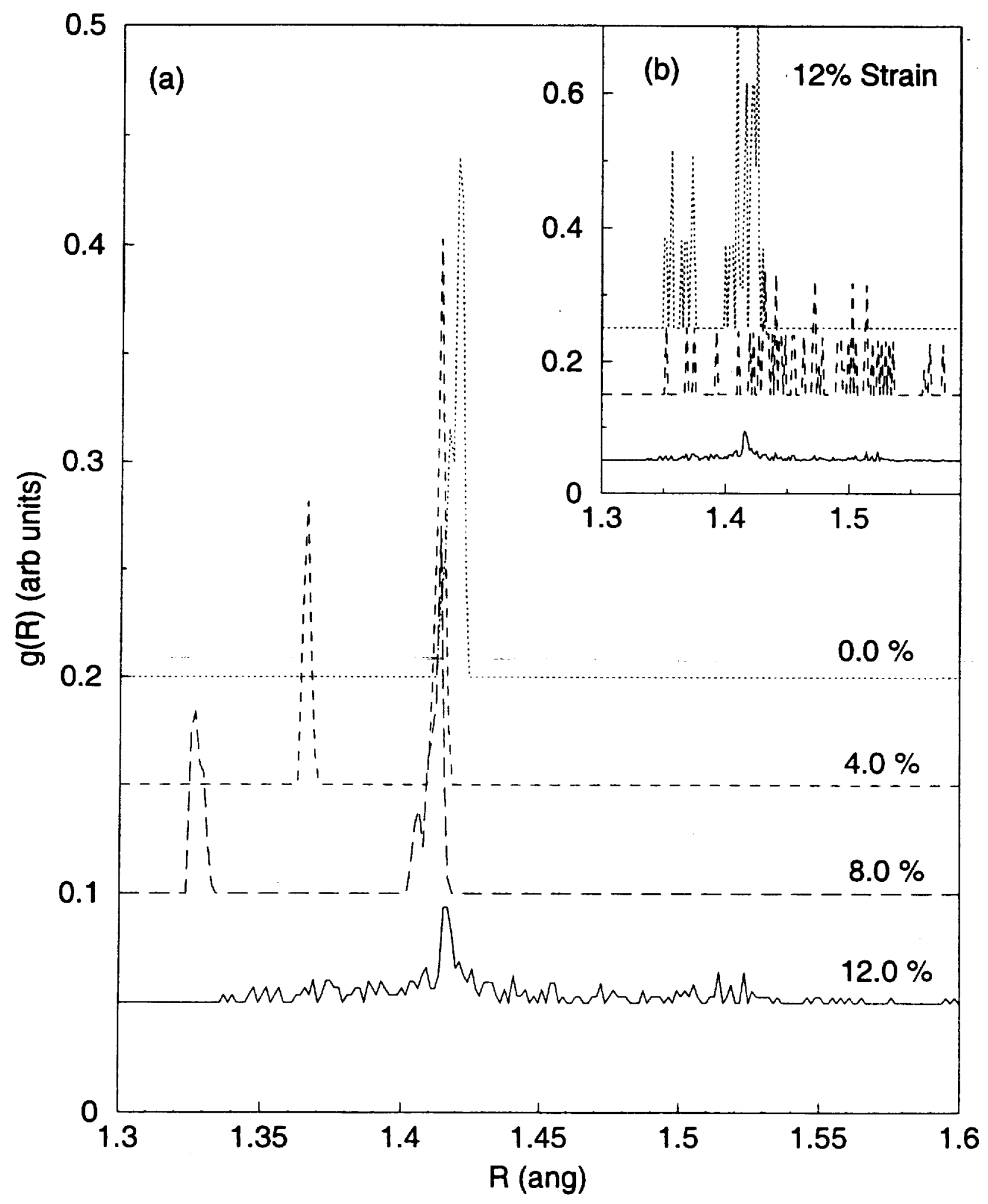

